\title{
Gravity research on plants: use of single-cell experimental models
}

\section{Youssef Chebli and Anja Geitmann*}

Département de Sciences Biologiques, Institut de Recherche en Biologie Végétale, Université de Montréal, Montréal, OC, Canada

\section{Edited by:}

David Ehrhardt, Stanford University, USA

\section{Reviewed by:}

Patrick H. Masson, University of

Wisconsin-Madison, USA

John Z. Kiss, Miami University, USA

*Correspondence:

Anja Geitmann, Département de

Sciences Biologiques, Institut de Recherche en Biologie Végétale,

Université de Montréal, 4101 Rue

Sherbrooke Est, Montréal, QC,

Canada H1X 2B2.

e-mail: anja.geitmann@umontreal.ca
Future space missions and implementation of permanent bases on Moon and Mars will greatly depend on the availability of ambient air and sustainable food supply. Therefore, understanding the effects of altered gravity conditions on plant metabolism and growth is vital for space missions and extra-terrestrial human existence. In this mini-review we summarize how plant cells are thought to perceive changes in magnitude and orientation of the gravity vector. The particular advantages of several single-celled model systems for gravity research are explored and an overview over recent advancements and potential use of these systems is provided.

Keywords: gravity, gravitropism, gravimorphogenesis, hyper-gravity, micro-gravity, pressure model, statolith, statocyte

\section{INTRODUCTION}

Long term space missions will greatly depend on the availability of ambient air, sustainable food supply, and treatment of human waste, all of which can be enhanced and improved through the cultivation of plants on-board the space craft (Musgrave, 2007; Wheeler, 2010). Plants also provide calming effects and emotional benefits that can be pivotal in the confined environment of a space craft or orbital platform as they help astronauts to fight loneliness and depression. The positive psychological effects on the crew have the potential to reduce stress resulting from the living and working conditions during a mission (Williams, 2002; Zimmermann, 2003). Because of their multiple roles, plants will play a primordial role in future space missions and understanding the plant metabolic and morphogenetic responses to altered gravity conditions is indispensable for the development of space craft ecosystems or long term planetary colonization at the fractional gravity levels found on the Moon (1/6 Earth's $g$ ) or Mars (3/8 Earth's $g$ ).

Cultivation of plants on orbital platforms affects growth of organs and individual cells as was shown in many plant species (Cowles et al., 1984; Kuang et al., 1996; Wolverton and Kiss, 2009; Matsumoto et al., 2010) although in many of these experiments the observed phenomena were a result of the combination of the direct effect of the absence of gravity on the plant and of other environmental factors such as increased radiation or absence of convection. Unlike most biotic and abiotic types of stress which plants have been exposed to during their evolution, gravity is the only constant factor, both in direction and magnitude, to which plants had to adapt in a permanent manner. To withstand the mechanical load imposed by gravity on terrestrial organisms, plants developed mainly two strategies. The first is based on the generation of a hydroskeleton which creates an erectile force based on the balance between the internal turgor pressure and the mechanical constraint by a highly tensile resistant extracellular matrix, the cell wall. The second is based on the fortification of the cell wall through hardening that even in the absence of internal turgor allows the individual cells to stay upright against the effect of compressive forces caused by gravity (Volkmann and Baluska, 2006). Modification of cell wall composition is, therefore, a readily observed phenomenon in plants exposed to a change in $g$-force (Waldron and Brett, 1990). This type of response has been termed gravity resistance (Hoson and Soga, 2003). Experiments that are performed to study these architectural responses of plants to the effect of $g$-force are generally based on increasing its magnitude through placing the specimen into a centrifuge, or by decreasing it through exposure to omnilateral or true micro-gravity conditions (Hemmersbach et al., 1999; Hoson and Soga, 2003; Figures 1A,B). Omnilateral micro-gravity conditions can be produced in a clinostat or a random positioning machine by turning the specimen in rotary 2D motion or randomly in 3D, either at slow or rapid speed (Skagen and Iversen, 1999; Figure 1C). This does not actually alter the magnitude of the gravity force but it minimizes the effect associated with a unidirectional stimulus. True micro-gravity conditions can be achieved either on orbital platforms or during the free fall phase of sounding rocket and parabolic flights.

Plants do not only respond to a change in magnitude but also to a change in orientation of the $g$-vector. These responses are termed gravimorphogenetic and are typically expressed in form of a gravitropic behavior that is oriented in the direction of the $g$ vector (positive gravitropic) or opposed to it (negative gravitropic; Kiss, 2000; Hoson and Soga, 2003). Typical responses include the re-orientation of the root and the shoot of a plant that has been turned on its side. Numerous studies have investigated these gravitropic responses both in multicellular organisms and single-cells (Baluska and Hasenstein, 1997; Braun and Sievers, 2000; Kiss, 2000; Perbal and Driss-Ecole, 2003; Morita, 2010). Typical experiments include simple changes in the direction of the gravity 
vector by reorienting the sample (Figure 1D). However, these experiments are frequently also conducted under micro- or hypergravity conditions to enhance the response and analyze dose-effect relationships. Intriguingly, in multicellular organisms, the gravitropic response typically occurs at a location that is spatially separated from the cells that are responsible for the perception of the directional signal and thus requires long-distance signaling processes about which our understanding has significantly

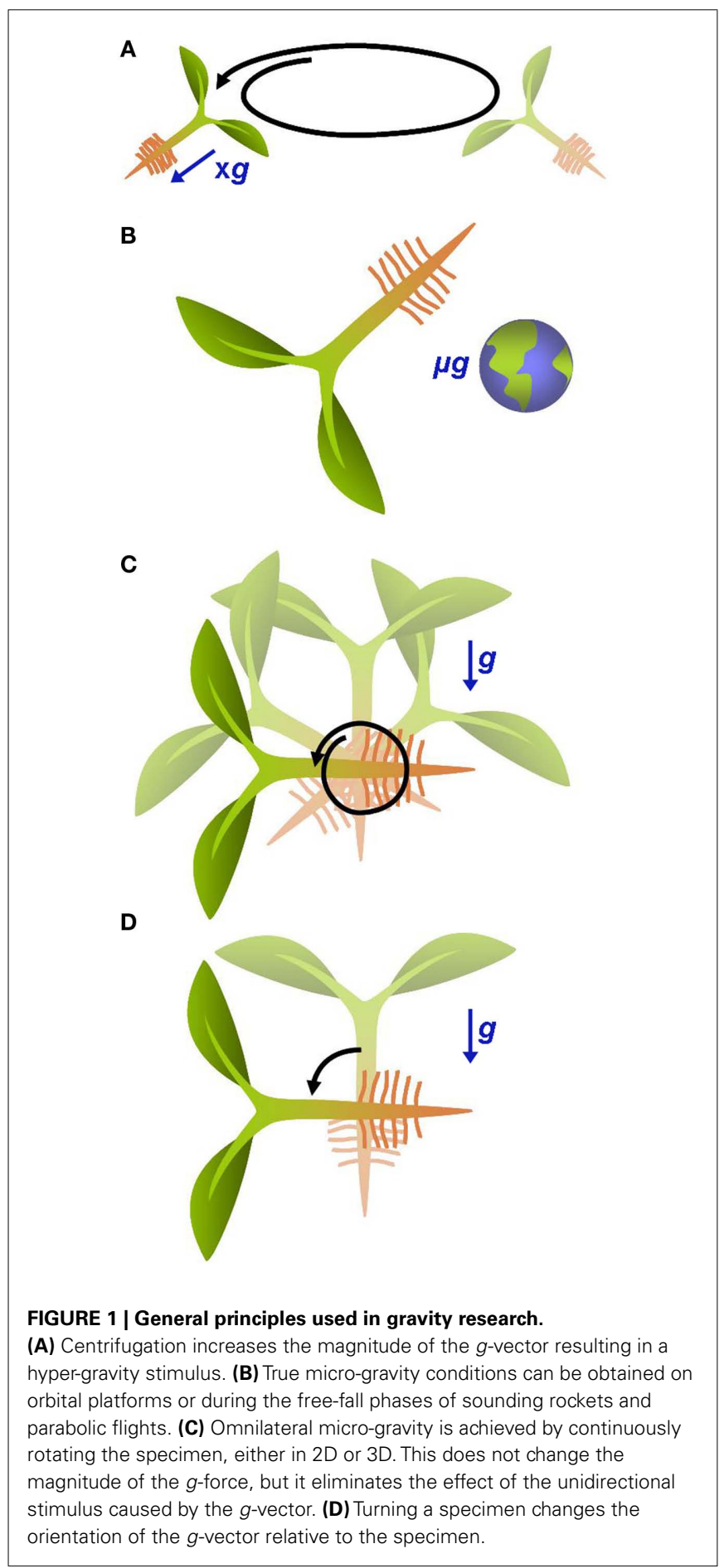

improved in recent years (Haswell, 2003; Morita, 2010). Auxin is an important mediator of gravity response in roots and shoots (reviewed by Morita, 2010). Gravitropism in Arabidopsis roots is controlled by basipetally transported auxin (Rashotte et al., 2000). Consistent with the important role of the hormone, transcriptomic studies have shown that the expression of genes related to auxin biosynthesis is altered by a change in gravity level (Tamaoki et al., 2011). Several auxin transporters from the PIN and PGP families are known to be involved in the distribution and the direction of auxin fluxes. The involvement of auxin and auxin transporters in gravisensing and graviresponse emphasize the importance of investigations into the regulatory mechanisms of the action of this hormone. For a thorough and up-to-date overview of auxin signaling, we refer to recently published reviews (Zhao, 2010; Wu et al., 2011).

The challenges of cultivating plants or plant cells at microor hyper- $g$ are manifold ranging from the complexity and spatial limitations of experimental setups in space flight conditions and centrifuges (Musgrave, 2007) to the limited time of exposure that is possible during sounding rocket (duration of 10-12 min) and parabolic flight experiments (duration of tens of seconds; Luttges, 1992). The limited duration of these experimental setups highlights the advantage of biological systems that respond within the given time frame of the respective experimental device. While intracellular signaling cascades are triggered within $1 \mathrm{~s}$ upon the perception of an external mechanical signal (Hejnowicz et al., 1998), metabolic cellular responses in most plants can take up to several hours or days to be measurable thus providing a critical lower time limit for the duration of experimentation (Dutcher et al., 1994; Mullen et al., 2000).

While using entire plants is necessary to study the effects on plant growth, architecture, and reproduction, studies on cellular metabolism can potentially take advantage of single-cell experimental systems. These have the advantage of being easier to observe microscopically and other experimental conditions are easier to control. In the present review we present several singlecell plant systems that have been used in the past years and that present great potential for gravity research, in particular for the investigation of the effects of gravity on plant cellular functioning and metabolism. To introduce the open questions in this field of research, it is worth summarizing how plant cells are thought to perceive the orientation and magnitude of the gravity vector. Several conceptual models have been proposed on how plant cells perceive gravity stimulation.

\section{CONCEPTS OF CELLULAR GRAVISENSING IN PLANTS STATOLITH-BASED GRAVISENSING}

In the statolith-based model, the gravity signal is triggered by the movements of small bodies inside the cytoplasm that are of higher density than the surrounding cytosol - the statoliths. The cells equipped with such statoliths are called statocytes. Statoliths typically consist of starch-containing amyloplasts or crystals such as those made of barium sulfate found in Chara rhizoids (Sievers et al., 1996; Kuznetsov et al., 2001; Perbal and Driss-Ecole, 2003). A change in the orientation of the gravity vector relative to the orientation of the organism causes the statoliths to sediment toward the new downward facing side of the cell and their movement results 
in the deformation of other sub-cellular structures (Figure 2A). It was thought for a long time that the moving particles exert a tensile stress on actin arrays which in turn influence the activity of membrane located mechano-sensitive ion channels (Baluska and Hasenstein, 1997; Sack, 1997; Hejnowicz et al., 1998; Morita and Tasaka, 2004). However, drug-induced disruption of the actin arrays enhances the gravity response in the roots of Arabidopsis and rice (Staves, 1997; Hou et al., 2004, 2003) as well as in Arabidopsis inflorescence stems and hypocotyls (Yamamoto and Kiss, 2002). Moreover, Arabidopsis mutants with reduced levels of starch-content are nevertheless able to perceive gravity signals (reviewed by Morita, 2010). Rather than the sedimenting motion it may therefore be the direct contact of amyloplasts with the endoplasmic reticulum (ER) located in the periphery of the cell that triggers the signal (Zheng and Staehelin, 2001; Perbal and DrissEcole, 2003; Morita, 2010). High resolution electron tomography has revealed that the force of gravity on the mass of statoliths is sufficient to locally deform the membranes of the cortical ER (Leitz et al., 2009). Clear evidence for the action of statoliths was provided by the fact that magnetophoretic displacement of statoliths in roots and shoots of higher plants as well as Chara rhizoids was able to induce gravitropic curvature (Kuznetsov and Hasenstein, 1996, 1997, 2001; Weise et al., 2000). These studies highlight the usefulness of micromanipulator strategies for gravitational research (Geitmann, 2006a,b, 2007). While a local membrane bending by statoliths was proposed to act in gravisensing of root columella cells (Leitz et al., 2009), it has been shown that in moss protonemata, statoliths do not need to exert pressure. The simple contact with a receptor located at the susceptible membrane (in this case the plasma membrane) suffices to elicit the response (Limbach et al., 2005). Whatever the precise biochemical signaling pathway will turn out to be, according to the statolith-based gravisensing model the cellular response depends on the intracellular motion or displacement of some sort of particle or organelle that has a higher density than the surrounding cytoplasm and that therefore sediments to the lowest region of the cell upon the re-orientation of the latter relative to the gravity vector.

\section{THE GRAVITATIONAL PRESSURE MODEL}

Most plant cells are not equipped with statoliths and a second type of gravity perception mechanism needs to be in place to explain the fact that these cells nevertheless respond to changes in $g$-force. Evidence for the presence of an alternative mechanism stems from studies on mosses, fungi, and algae which show gravity-dependent growth and differentiation without the presence of statoliths (Staves, 1997). In higher plants as well, a statolith-independent pathway seems to operate. Carefully adjusted rotation of roots that maintains the statolith-equipped root cap vertical during gravitropic bending does not cause the root to abolish the bending process. This supports the view that there is a second, statocyteindependent location in the root where the gravistimulus is perceived (Wolverton et al., 2002). While at times considered a controversy, it became clear that several mechanisms of gravisensing seem to operate, possibly even in the same cell (Barlow, 1995; Sack, 1997; Hasenstein, 1999; Kiss, 2000). The presence of an alternative mechanism can explain why Arabidopsis mutants unable to synthesize starch display an operating gravitropic response in roots
A

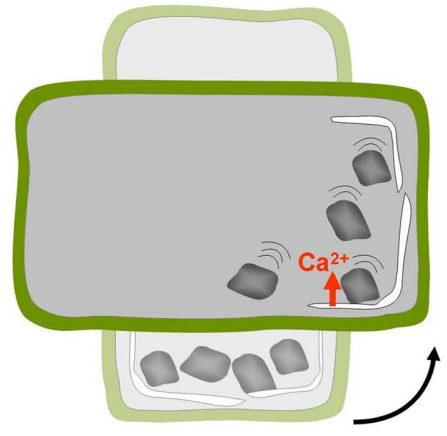

B

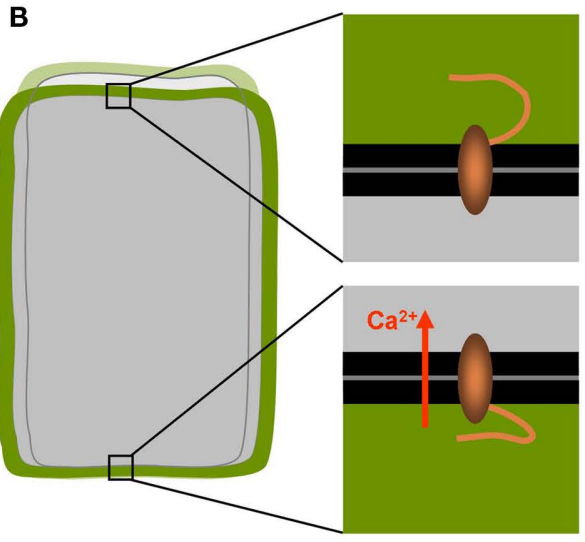

C

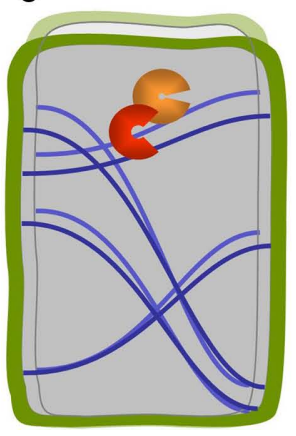

D

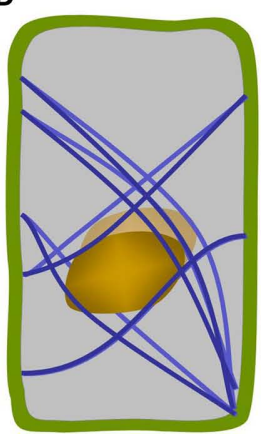

FIGURE 2 | Concepts of cellular gravisensing in plants. (A) In

statolith-based gravisensing the sedimentation or change of position of intracellular organelles with higher density triggers a signal most likely based on a change in trans-membrane ion fluxes. (B) According to the gravitational pressure model the weight of the protoplast causes the forces acting on the membrane-cell wall connections at the upper and lower sides of the cells to be different. (C) The tensegrity model predicts that cellular distortion due to a change in $g$-force affects the pre-stress in the cytoskeletal array of the cell which in turn changes biochemical activities. (D) A variation of the tensegrity model is based on a change in cytoskeletal pre-stress being caused by the weight of heavy organelles that are tethered to the cytoskeletal filaments such as the nucleus.

and shoots albeit diminished (Caspar et al., 1985; Hatakeda et al., 2003; Soga et al., 2005; Buizer, 2007). The gravitational pressure model provides a possible but not necessary the only explanation for this phenomenon. It suggests that the entire mass of the protoplast acts as a gravity sensor that behaves similar to a waterfilled balloon that flattens when placed on a surface due to its own 
weight. In this model the role of starch-filled amyloplasts would be that of increasing the overall density of the protoplast (Wayne and Staves, 1996). It is postulated that membrane proteins located at the top and bottom of cell may be activated through the action of differential tensile forces as they interact with the lower and upper cell walls, respectively (Wayne et al., 1992; Wayne and Staves, 1996; Figure 2B). Hitherto, the gravitational pressure model has been based on experimentation on the internodal cells of Characean algae and its relevance for graviperception in higher plants has yet to be demonstrated.

\section{TENSEGRITY MODEL}

An alternative, but not mutually exclusive view, on how deforming forces acting on the cell as a whole could be perceived, is through the effect of cellular distortion on the mechanics or flexibility of the cytoskeletal arrays as explained by the tensegrity model of cell functioning. Although this model was developed for animal cells (Ingber, 1999), it could doubtless be transferred to plant cells. In this model, the exposure to micro-gravity decreases the internal pre-stress in the cytoskeletal array consisting of elements that resist compressive (microtubules) and tensile stresses (actin filaments). This conceptual model of cell functioning is based on the architectural principles by Buckminster Fuller (Ingber, 1993). The distortion induced change in preexisting force balance is supposed to affect local thermodynamic or kinetic parameters and thus biochemical activities (Figure 2C). How mechanical signals perceived at the cell surface could influence intracellular processes has been reviewed in detail (Ingber, 2006; Orr et al., 2006). However, the change in pre-stress of the cytoskeletal arrays does not necessarily need to be caused by the distortion of the outer shape of the cell but could also result from the gravity force acting on organelles attached to this network (Yang et al., 2008; Figure 2D). Finite element modeling has shown that the difference in density between the nucleus and the cytoplasm would be sufficient to cause a change in tensile stress that is significant enough to deform the cytoskeletal array upon application or removal of gravity (Yang et al., 2008).

While according to the tensegrity model the activities of numerous cytoplasmic enzymes are affected directly, the crucial mechanotransduction step in the statolith and pressure models is the deformation of a membrane (plasma membrane, tonoplast, mitochondria, ER) which in turn influences trans-membrane $\mathrm{Ca}^{2+}$ fluxes and consequently the cytosolic concentration of the ion (Fasano et al., 2002; Toyota et al., 2008). Although in statocytes and gravitactic unicellular organisms, calcium was shown to be involved in graviperception as well as in signaling processes leading to a graviresponse, in non-statocyte cells in higher plants, knowledge on calcium signaling involvement in graviresponse is scant (Sinclair and Trewavas, 1997; Kordyum, 2003), and warrants further investigation.

\section{SINGLE-CELL SYSTEMS USEFUL FOR UNDERSTANDING STATOLITH-INDEPENDENT GRAVIPERCEPTION}

Immediate and short-term responses can be difficult to assess in multicellular systems, and therefore the use of single-cells in culture has been a successful alternative approach that complements our understanding of graviperception and responses in plants. Handling of single-cells is generally easier (although typically sterile conditions have to be ensured) and depending on the parameter of interest, the response can often be observed shortly after application of the environmental trigger. Moreover, microscopy is facilitated since no neighboring cells obstruct the view, thus allowing for high spatial and temporal resolution imaging. Finally, reproducible growth conditions are more readily achieved in cell cultures since parameters such as temperature, nutrient concentrations, and $\mathrm{pH}$ can be tightly controlled.

Among the most intensively studied single-cell systems investigated in micro-gravity research are the protonemata and rhizoids of mosses and Chara, a freshwater alga. Although these cells are part of a multicellular organism, they are tip-growing individual cells which makes them readily accessible to microscopic observation. Furthermore, gravity perception and response occur in the same cell. Both protonemata and rhizoids are equipped with statoliths and important information has been gained from numerous studies on these organisms which have been exhaustively reviewed (Schwuchow et al., 1990, 1995; Sievers et al., 1996; Braun, 1997; Sack, 1997; Demkiv et al., 1999; Braun and Sievers, 2000; Braun and Wasteneys, 2000; Braun and Limbach, 2006). In the following, we will confine our overview to studies performed on single plant cell systems that are not equipped with statoliths and that offer the possibility to assess how gravity or the absence thereof affect basic plant cell functioning and metabolism.

\section{CELL WALL ASSEMBLY IN PROTOPLASTS}

The molecular architecture of the cell wall and, by consequence, its mechanical behavior are known to be affected in many but not all plants grown under micro- and hyper-gravity conditions (Cowles et al., 1984; Waldron and Brett, 1990; Nedukha et al., 1994; Hoson et al., 1996; Nedukha, 1996; Soga et al., 1999a,b, 2001; Levine et al., 2001). Therefore, the investigation of altered $g$-force on the kinetics of the cell wall assembly process is of considerable interest. Single-cell studies to this end have been conducted on protoplasts generated from different plant systems. After the enzymatic removal of the cell wall, protoplasts generally start to regenerate a new cell wall. This is followed by cell division and formation of small cell aggregates few days later. These aggregates develop into callus tissue and, under suitable conditions, into mature plants. It is in particular the ability to regenerate mature plants from protoplast cultures that makes this experimental system interesting for applications in space exploration. Under micro-gravity conditions, cell wall formation in protoplasts isolated from Brassica napus, Daucus carota, and Solanum tuberosum is significantly delayed compared with the control samples at $1-g$ (Nedukha et al., 1994; Rasmussen et al., 1994, 1992). In particular, the content of structural components such as cellulose and hemicellulose is reduced whereas pectin is unaltered (Nedukha, 1998; Skagen and Iversen, 2000). These micro-gravity induced delays in protoplast regeneration also slow callus formation but do not prevent the process. However, under micro- $g$ conditions development of intact plants is hampered, since calluses develop either roots or shoots, but not both (Iversen et al., 1999).

In these studies, peroxidase activity was measured in the regenerating protoplasts and revealed a decrease in enzyme activity compared to the ground control (Rasmussen et al., 1992). Since 
peroxidase activity is involved in cell wall metabolism and crosslinking of microfibrils, this reduced activity was proposed to provide a possible explanation for the observed slow-down in cell wall regeneration. However, later experiments showed that factors other than weightlessness (such as cosmic radiation) may have contributed to or even caused this change in peroxidase activity (Skagen and Iversen, 2000).

Interestingly, another cellular feature may be the reason for retarded cell wall deposition, in particular reduced cellulose deposition: the cortical microtubule cytoskeleton. The amount of cortical microtubules at $24 \mathrm{~h}$ after protoplast isolation is greater in protoplasts cultured at 1- $g$ than under micro-gravity (Skagen and Iversen, 2000). Moreover, while cortical microtubules assessed at $24 \mathrm{~h}$ after cell wall removal in recovering rapeseed protoplasts are organized in parallel arrays, they are randomly oriented in the $0-g$ sample and hence unaltered from those of the protoplast immediately after cell wall removal. Since cortical microtubules play an important role in cellulose microfibril deposition (Emons et al., 2007), the failure to reorganize may certainly influence the capacity of the cell to fully regenerate its wall, and subsequently, to divide.

\section{CALCIUM FLUXES IN POLAR FERN SPORES}

One of the single-cell systems capable of a gravitropic response is the gametophyte of the fern Ceratopteris richardii. During the first $24 \mathrm{~h}$ of germination, the gravity vector determines the axis of development of the spore by setting an asymmetrical growth polarity creating two asymmetrical cells that will grow parallel to gravity in opposite directions (Edwards and Roux, 1998; Chatterjee and Roux, 2000). When developed in micro-gravity conditions, the spores are able to germinate but lose the spatial polarity (Roux et al., 2003). The same pattern was observed when calcium channel blockers or inhibitors such as nifedipine and eosin yellow were added to the culture medium after germination initiation (Chatterjee et al., 2000). Efflux of calcium at different cellular regions was recorded using ion selective electrodes on spores grown at $1-g$ and spores grown during parabolic flights (the $g$ level fluctuated between micro- $g$ and $1.8-g$ ). The results revealed that the specific activation of mechano-sensitive calcium channels at the bottom of the cell is required for graviperception (Chatterjee and Roux, 2000; Salmi et al., 2011). These data confirm the notion that calcium fluxes are involved in graviperception in multicellular plants (Sinclair and Trewavas, 1997; Fasano et al., 2002; Soga et al., 2002; Hoson et al., 2010). Use of the fern spore system allowed for facilitated microscopical access and measurement of ion fluxes compared to the multicellular root cell systems. However, the mechanism by which the channels are activated and the pathways that are involved in the transduction of the gravitydependent response are yet to be determined. Progress on the understanding of the molecular mechanism will certainly profit from advances made on other mechanoperceptive cellular systems (Orr et al., 2006; Poirier and Iglesias, 2007).

\section{MICROTUBULE CYTOSKELETON IN BY-2 CELLS}

The microtubule cytoskeleton has multiple functions in plant cells including the guidance of the intracellular motion of organelles, the targeting of enzymes involved in cell wall assembly, chromosome separation, and cell plate formation during mitosis.
When and where microtubules are assembled from tubulin and disassembled, therefore, is pivotal for cellular functioning. In weightlessness, isolated tubulin does not self-organize into parallel microtubule bands as it does in the same in vitro conditions on the ground (Papaseit et al., 2000). Similarly, in different types of cultured mammalian cells, the organization of the microtubule cytoskeleton is affected under real or omnilateral micro-gravity conditions (Lewis et al., 1998; Rösner et al., 2006). The finding that microtubules in regenerating protoplasts cultured under microgravity conditions were less organized than in the ground control provided a motivation for investigating the microtubule cytoskeleton in cultures of other single plant cell types. Similar to the protoplasts, microtubules in cultured cells dedifferentiated from tobacco hypocotyls at $1-g$ were more abundant than their counterpart grown in micro-gravity (Sato et al., 1999). The microtubules of a third single-celled system, tobacco BY-2 cells, were found to be less susceptible to a change in $g$-force, on the other hand. The BY-2 cell line was established from a callus induced on a seedling of Nicotiana tabacum cultivar Bright Yellow-2. Tobacco BY-2 cells grow rapidly in suspension culture and can multiply their numbers up to 100 -fold within 1 week in adequate culture medium. Remarkably, exposure of BY-2 cells to micro-gravity conditions did not have any effect on cell division or cell growth. The organization of cortical microtubules was identical to that in cells cultured on Earth, and the orientation of newly deposited cellulose microfibrils was unaltered (Sieberer et al., 2009). Therefore, the tissue context does not seem to be a prerequisite for these cells to ensure cytoskeletal ordering under weightlessness. Rather it seems that the absence of a cell wall hampers microtubule organization during the initial phase of protoplast regeneration. This is consistent with the observation that microtubules in plant cells tend to align parallel to the principal direction of stress and thus respond to mechanical cues (Hush and Overall, 1991; Geitmann et al., 1997; Hamant et al., 2008). In line with this, the expression of tubulin genes is upregulated by hyper-gravity in Arabidopsis hypocotyls (Matsumoto et al., 2007) and cortical microtubules are reoriented from transverse to longitudinal (Soga et al., 2006). In tubulin mutants of Arabidopsis displaying twisted growth, hyper-gravity caused a more pronounced twisting phenotype and microtubule re-orientation was more prominent (Matsumoto et al., 2010). This led to the hypothesis that by influencing cellulose deposition, microtubules play an important role in the maintenance of a normal growth phenotype against the gravitational force (Hoson et al., 2010).

\section{ENDOMEMBRANE TRAFFICKING IN THE POLLEN TUBE}

Experiments placed on parabolic flight and sounding rockets only make sense if the organism or cell displays a response to microgravity that appears within the short duration of weightlessness achieved during the flight. Assessment of both cellular growth and metabolism using these devices can therefore be exploited with cell systems in which both parameters are highly active and change rapidly upon exposure to a trigger. The fastest growing plant cell is the pollen tube, a cellular protrusion formed by the pollen grain upon contact with the receptive stigma. The pollen tube is responsible for fertilization in higher plants and hence crucial for seed and fruit formation. Pollen tubes, like protonemata, rhizoids, root hairs, fungal hyphae, and neurons, are tip-growing 
cells. The growth rates of pollen tubes can be up to hundreds of micrometers per minute and sustaining this process requires the continuous deposition of cell wall material in highly controlled manner to ensure morphogenesis of a perfectly cylindrical shape (Figure 3).

The development of the pollen grain, or microgametophyte, occurs in the anther and comprises several, precisely regulated cell divisions (meiotic and mitotic), the assembly of a highly structured and extremely resistant cell wall, and the coordinated activation of the cytoskeleton (Honys et al., 2006; Bou Daher et al., 2011; Liu et al., 2011). Long term studies on orbital platforms have shown that pollen formation was aborted at early stages and young microspores were deformed and empty. In late developmental stages, the pollen exine was able to form but the cytoplasm seemed contracted and became disorganized (Kuang et al., 1995). However, rather than an effect of micro-gravity, these phenomena were found to be a consequence of the reduced carbon dioxide environment due to lack of convective air movement. When plants were grown in high $\mathrm{CO}_{2}$ atmosphere, pollen with normal outer morphology could be obtained from Arabidopsis and Brassica. However, although the percentage of viable pollen was much higher than during the earlier experiment, fertilization did not occur (Kuang et al., 1996). Optical and electron microscopy showed that pollen grains developed in micro-gravity displayed differences in size, shape, number of mitochondria as well as an abundant presence of large starch grains absent in the pollen that developed on the ground (Kuang et al., 1995, 2005). When environmental conditions at micro- $g$ such as $\mathrm{CO}_{2}$, light and convection were more carefully controlled, it was possible to obtain viable embryos, seeds, and siliques in Arabidopsis and Brassica proving that pollen tube growth was indeed possible at micro- $g$ (Musgrave et al., 1997; Popova et al., 2009).

At hyper-gravity the effects on pollen and fertilization vary significantly between species. While at $4-g$ seed set in Brassica was not affected (Musgrave et al., 2009a), the formation of siliques was significantly reduced in Arabidopsis (Musgrave et al., 2009b). The authors found this to be due to a reduced ability of Arabidopsis pollen tubes to germinate at $4-g$. They propose that the increasing $g$-force caused the cytoplasm to exert higher than normal pressure on the cell wall disturbing the tip growth process (Musgrave et al., 2009b). The main metabolic activity of the pollen tube is the synthesis and the deposition of cell wall precursors which are indispensable for the continuing assembly of the elongating cell (Geitmann and Steer, 2006). The principal phenomenon responsible for the tube expansion is the exocytosis of vesicles containing pectins which occurs at high rates and is spatially and temporally regulated by a multitude of parameters (Chebli and Geitmann, 2007). Other cell wall components such as cellulose, xyloglucans, and callose are either deposited by exocytosis or directly synthesized at the plasma membrane. Each of these components plays

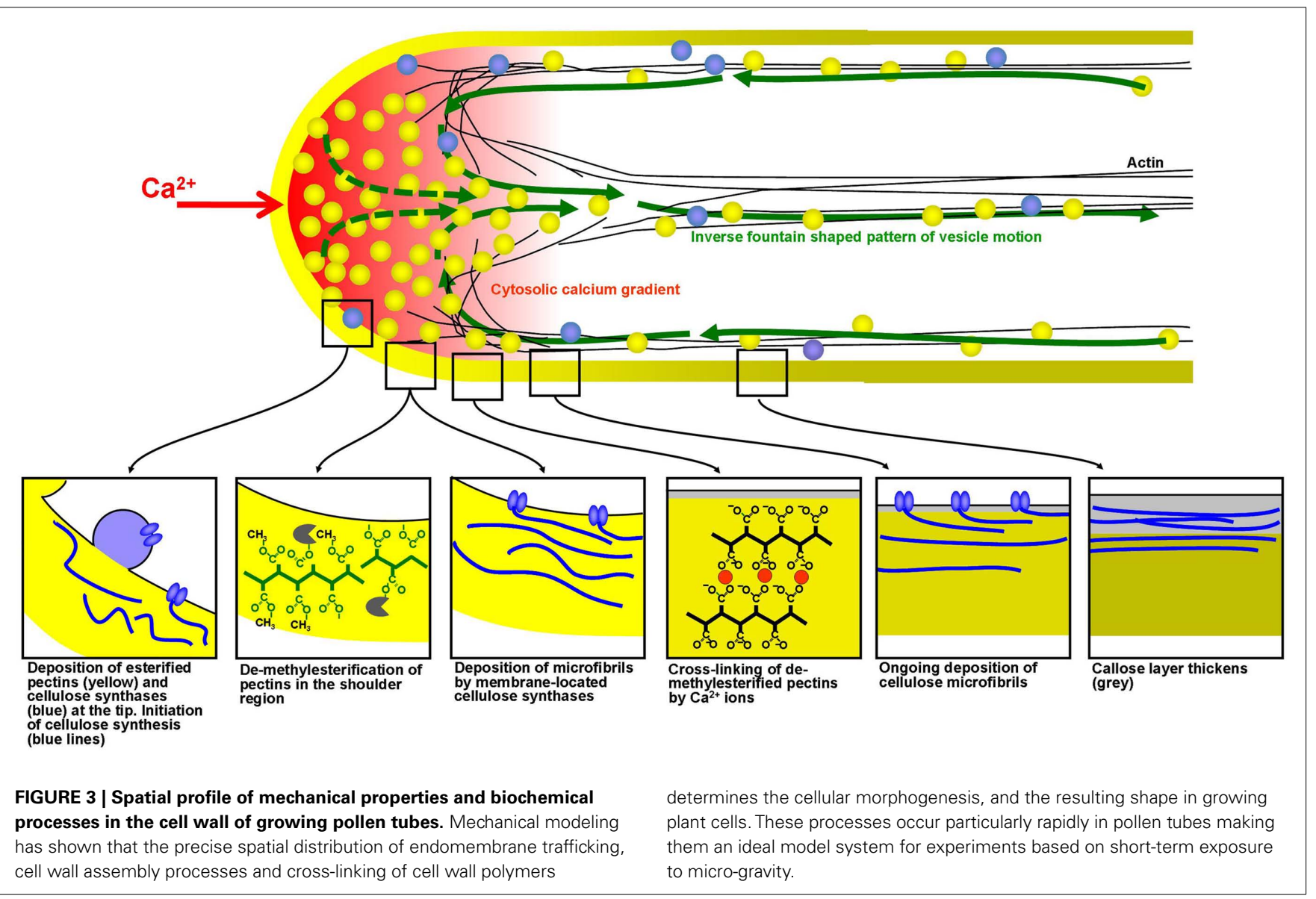




\begin{abstract}
Glossary
Gravimorphogenetic

Graviperception

Graviresponse

Gravisensing

Gravistimulus

Gravitropism

Gravity resistance

Mechanotransduction

Omnilateral

Statocyte

Statolith

Developmental change in response to the presence or the change in the orientation or magnitude of the $g$-vector

The ability of a cell to become aware of the orientation or magnitude of the gravity vector

Physiological or morphogenetic response of a cell or a tissue to gravity induced trigger. The responding cell does not need to be identical to the perceiving cell

Perception of gravity stimulus

Mechanical process caused by the presence or change in magnitude or orientation of gravity

Ability of a plant, organ or cell to orient its growth in the direction of the gravity vector

Gravimorphogenetic response that serves to reinforce the organism or organ against the effect of gravity. Typically a reinforcement of the cell wall

Conversion of a mechanical stimulus into a chemical intracellular signaling pathway

From all sides

Cell specialized in graviperception and typically equipped with statoliths

Intracellular body with density higher than the surrounding cytosol causing the body to sediment in the direction of the gravity vector. Either the sedimenting motion or the new position of the statolith provides the cell with information on magnitude and orientation of the gravity vector
\end{abstract}

a defined mechanical role during pollen tube growth and in each plant species these components are distributed differently forming a characteristic spatial profile (Geitmann and Dumais, 2009; Fayant et al., 2010; Figure 3). Intriguingly, the pollen tube is able to compensate for the lack of one of the components by overproduction of another (Aouar et al., 2010), demonstrating that sophisticated mechanical control mechanisms must be in place to ensure that the final product is functional.

Very conveniently, pollen tubes are easily cultured in vitro thus allowing high resolution microscopic observations. Crucial in the present context, the high growth rate entails rapid and easily visible cellular responses upon mechanical or chemical manipulation (Geitmann and Steer, 2006; Chebli and Geitmann, 2007). The responses of in vitro growing pollen tubes developing under micro- $g$ conditions or during clinostat rotation vary between plant species. In Prunus persica pollen tubes grown in a clinostat, callose plugs were four times longer than those in the control tubes and callose was spread along throughout the tube (De Micco et al., $2006 a, b)$ indicating that cell wall assembly under altered gravity conditions is affected. Unlike somatic plant cells in which an altered cell wall assembly clearly fulfills a structural purpose since it reinforces the cell wall against the mechanical stress to which it is exposed, the performance of a pollen tube would not really be improved by a stiffer cell wall. The cellular response is therefore likely unspecific and offers the unique possibility to study the fundamental effect of altered gravity conditions on plant cell metabolism.

Cell wall assembly in the pollen tube is ensured by a high rate of intracellular trafficking targeted toward the growing end of the cell (Bove et al., 2008; Kroeger et al., 2009; Bou Daher and Geitmann, 2011). Temporal and spatial control of the vesicle fusion responsible for cell wall assembly at the growing tip is required to determine the rate and direction of growth (Fayant et al., 2010). The influx of calcium through plasma membrane located calcium channels at the tip of the cells plays an important role in the temporal and spatial regulation of the growth process (Hepler, 1997; Feijó et al., 2001; Chebli and Geitmann, 2007). Exocytosis in the tip region is accompanied by membrane endocytosis to ensure a balanced deposition rate of cell wall and membrane material. The delicate equilibrium between exocytosis and endocytosis is disturbed when in vitro growing pollen tubes are exposed to micro- and hyper- $g$ conditions. In micro-gravity, the uptake of a fluorescent phospholipid into pollen tubes, an indication for endocytosis, is increased, whereas the contrary is true for hyper-gravity conditions (Lisboa et al., 2002). Since pollen tubes are not gravitropic (De Micco et al., 2006b), they can therefore serve as model systems that allow the investigation of statolith-independent, non-gravitropic responses of plant cells upon altered gravity conditions. Moreover, understanding the performance of this cell is crucial for future applications in space flight as successful fertilization is essential for on-board production of plant-based food.

\section{CONCLUSION}

Experiments based on entire plants are inevitable to understand plant functioning under altered gravity conditions. Nevertheless, single-cell experimental systems have emerged as excellent tools that allow for in-depth studies of individual processes that are altered as a consequence of exposure to micro- or hyper-gravity conditions. It becomes increasingly clear that statolith mediated effects are not the only responses that contribute to plant behavior under altered gravity conditions. So far, each of the cellular systems mentioned above has been used to investigate particular aspects of cellular functioning. An important step forward will be the combined analysis of different processes on a single system to obtain a more holistic view of the involved mechanisms and to connect the dots. Secondly, identifying common principles shared by different cellular systems, as well as distinctive features that can be explained by the particular functionality of the cell wall will allow to make conclusions for general plant cell functioning. By way of example, cell wall regeneration was investigated in recovering protoplasts, but elongating tip-growing cells represent alternative systems that provides complementary information. The effect of gravity on the cytoskeleton has mostly been studied on comparably slowly developing cells such as BY-2 cells, but important information could probably gained from and short-term experimental devices could be exploited for systems in which these cellular features are highly dynamic such as growing pollen tubes. Ion fluxes, so far only studied in fern spores in the context of gravity research, could, and should equally be studied in other growing cells in which ion flux profiles have been established. Single-cell 
systems allow for the use of imaging methods that operate at high spatial and temporal resolution and will therefore enable us to determine the roles of sub-cellular features such as targeted transport processes and ion fluxes that lead to a tropic response or a change in metabolic homeostasis. It will then be possible to link these results to those obtained from transcriptomic and proteomic approaches, thus making sense of the overwhelming wealth of data that these types of studies produce (Martzivanou and Hampp, 2003; Martzivanou et al., 2006; Wang et al., 2006; Babbick et al., 2007; Barjaktarovic et al., 2007, 2009). An integration of cell biological and imaging approaches with quantitative information on the expression of proteins involved in cell wall synthesis, lipid

\section{REFERENCES}

Aouar, L., Chebli, Y., and Geitmann, A. (2010). Morphogenesis of complex plant cell shapes - the mechanical role of crystalline cellulose in growing pollen tubes. Sex. Plant Reprod. 23, 15-27.

Babbick, M., Dijkstra, C., Larkin, O., Anthony, P., Davey, M., Power, J., Lowe, K., Cogoli-Greuter, M., and Hampp, R. (2007). Expression of transcription factors after short-term exposure of Arabidopsis thaliana cell cultures to hypergravity and simulated microgravity (2-D/3-D clinorotation, magnetic levitation). Adv. Space Res. 39, 1182-1189.

Baluska, F., and Hasenstein, K. H. (1997). Root cytoskeleton: its role in perception of and response to gravity. Planta 203, S69-S78.

Barjaktarovic, Ž., Nordheim, A., Lamkemeyer, T., Fladerer, C., Madlung, J., and Hampp, R. (2007). Time-course of changes in amounts of specific proteins upon exposure to hyper-g, 2-D clinorotation, and 3-D random positioning of Arabidopsis cell cultures. J. Exp. Bot. 58, 4357-4363.

Barjaktarovic, Ž., Schütz, W., Madlung, J., Fladerer, C., Nordheim, A., and Hampp, R. (2009). Changes in the effective gravitational field strength affect the state of phosphorylation of stress-related proteins in callus cultures of Arabidopsis thaliana. J. Exp. Bot. 60, 779-789.

Barlow, P. W. (1995). Gravity perception in plants: a multiplicity of systems derived by evolution? Plant Cell Environ. 18, 951-962.

Bou Daher, F., and Geitmann, A. (2011). Actin is involved in pollen tube tropism through redefining the spatial targeting of secretory vesicles. Traffic. doi: 10.1111/j.16000854.2011.01256.x. [Epub ahead of print].

Bou Daher, F., van Oostende, C., and Geitmann, A. (2011). Spatial and temporal expression of actin depolymerizing factors
ADF7 and ADF10 during male gametophyte development in Arabidopsis thaliana. Plant Cell Physiol. 52, 1177-1192.

Bove, J., Vaillancourt, B., Kroeger, J., Geitmann, A. (2008). Magnitude and direction of vesicle dynamics in growing pollen tubes using spatiotemporal image correlation spectroscopy (STICS). Plant Physiol. 147, 1646-1658.

Braun, M. (1997). Gravitropism in tipgrowing cells. Planta 203, S11-S19.

Braun, M., and Limbach, C. (2006). Rhizoids and protonemata of characean algae: model cells for research on polarized growth and plant gravity sensing. Protoplasma 229, 133-142.

Braun, M., and Sievers, A. (2000). Plant cells on earth and in space. Korean J. Biol. Sci. 4, 201-214.

Braun, M., and Wasteneys, G. (2000). "Actin in characean rhizoids and protonemata. Tip growth, gravity sensing and photomorphogenesis," in Actin: A Dynamic Framework for Multiple Plant Cell Functions, eds C. Staiger, F. Baluska, P. Volkmann, and P. Barlow (Dordrecht: Kluwer Academic Publishers), 237-258.

Buizer, K. (2007). GraPhoBox: gravitropism and phototropism in Arabidopsis thaliana. Microgravity Sci. Technol. 19, 239-243.

Caspar, T., Huber, S. C., and Somerville, C. (1985). Alterations in growth, photosynthesis, and respiration in a starchless mutant of Arabidopsis thaliana (L.) deficient in chloroplast phosphoglucomutase activity. Plant Physiol. 79, 11-17.

Chatterjee, A., Porterfield, D. M., Smith, P. J. S., and Roux, S. J. (2000). Gravity directed calcium current in germinating spores of Ceratopteris richardii. Planta 210, 607-610.

Chatterjee, A., and Roux, S. J. (2000). Ceratopteris richardii: a productive model for revealing secrets of signaling and development. J. Plant Growth Regul. 19, 284-289. Hepler, P. K., Wiseman, P. W., and

metabolism, cell division, etc., will help to validate existing models and inform future models. Crucially, a multifaceted view will guide the development of new biomechanical and structural approaches to decipher the pathways of gravisensing and graviresponse.

\section{ACKNOWLEDGMENTS}

Research in the Geitmann lab is supported by grants from the Natural Sciences and Engineering Research Council of Canada (NSERC) and the Fonds Québécois de la Recherche sur la Nature et les Technologies (FQRNT). Youssef Chebli is funded by the Ann Oaks doctoral scholarship of the Canadian Society of Plant Physiologists.

Chebli, Y., and Geitmann, A. (2007). Mechanical principles governing pollen tube growth. Funct. Plant Sci. Biotechnol. 1, 232-245.

Cowles, J. R., Scheld, H. W., Lemay, R., and Peterson, C. (1984). Growth and lignification in seedlings exposed to eight days of microgravity. Ann. Bot. 54, 33-48.

De Micco, V., Scala, M., and Aronne, G. (2006a). Effects of simulated microgravity on male gametophyte of Prunus, Pyrus, and Brassica species. Protoplasma 228, 121-126.

De Micco, V., Scala, M., and Aronne, G. (2006b). Evaluation of the effect of clinostat rotation on pollen germination and tube development as a tool for selection of plants in space. Acta Astronaut. 58, 464-470.

Demkiv, O. T., Kordyum, E. L., Kardash, O. R., and Khorkavtsiv, O. Y. (1999). Gravitropism and phototropism in protonemata of the moss Pohlia nutans (hedw.) Lindb. $A d v$. Space Res. 23, 1999-2004.

Dutcher, F., Hess, E., and Halstead, T. (1994). Progress in plant research in space. Adv. Space Res. 14, 159-171.

Edwards, E. S., and Roux, S. J. (1998). Influence of gravity and light on the developmental polarity of Ceratopteris richardii fern spores. Planta 205, 553-560.

Emons, A., Höfte, H., and Mulder, B. M. (2007). Microtubules and cellulose microfibrils: how intimate is their relationship? Trends Plant Sci. 12, 279-281.

Fasano, J. M., Massa, G. D., and Gilroy, S. (2002). Ionic signaling in plant responses to gravity and touch. $J$. Plant Growth Regul. 21, 71-88.

Fayant, P., Girlanda, O., Chebli, Y., Aubin, C. E., Villemure, I., and Geitmann, A. (2010). Finite element model of polar growth in pollen tubes. Plant Cell 22, 2579-2593.

Feijó, J. A., Sainhas, J., Holdaway-Clarke, T. L., Cordeiro, M. S., Kunkel, J. G., and Hepler, P. K. (2001). Cellular oscillations and the regulation of growth: the pollen tube paradigm. Bioessays 23, 86-94.

Geitmann, A. (2006a). Experimental approaches used to quantify physical parameters at cellular and subcellular levels. Am. J. Bot. 93, 1220-1230.

Geitmann, A. (2006b). Plant and fungal cytomechanics: quantifying and modeling cellular architecture. Can. J. Bot. 84, 581-593.

Geitmann, A. (2007). Cytomechanical tools for plant gravitational biology. Gravit. Space Biol. 20, 31-42.

Geitmann, A., and Dumais, J. (2009). Not-so-tip-growth. Plant Signal. Behav. 4, 136-138.

Geitmann, A., Hush, J. M., and Overall, R. L. (1997). Inhibition of ethylene biosynthesis does not block microtubule re-orientation in wounded pea roots. Protoplasma 198, 135-142.

Geitmann, A., and Steer, M. W. (2006). "The architecture and properties of the pollen tube cell wall," in The Pollen Tube: A Cellular and Molecular Perspective, Plant Cell Monographs, ed. R. Malhó (Berlin: Springer Verlag), 177-200.

Hamant, O., Heisler, M., Jönsson, H., Krupinski, P., Uyttewaaal, M., Bokov, P., Corson, F., Sahlin, P., Boudaoud, A., Meyerowitz, E., Couder, Y., and Traas, J. (2008). Developmental patterning by mechanical signals in Arabidopsis. Science 322, 1650-1655.

Hasenstein, K. H. (1999). Gravisensing and plants and fungi. Adv. Space Res. 24, 677-685.

Haswell, E. S. (2003). Gravity perception: how plants stand up for themselves. Curr. Biol. 13, R761-R763.

Hatakeda, Y., Kamada, M., Goto, N., Fukaki, H., Tasaka, M., Suge, H., and Takahashi, H. (2003). Gravitropic response plays an important role in the nutational movements of the shoots of Pharbitis nil and Arabidopsis thaliana. Physiol. Plant. 118, 464-473.

Hejnowicz, Z., Sondag, C., Alt, W., and Sievers, A. (1998). Temporal course of graviperception in intermittently 
stimulated cress roots. Plant Cell Environ. 21, 1293-1300.

Hemmersbach, R., Volkmann, D., and Häder, D.-P. (1999). Graviorientation in protists and plants. J. Plant Physiol. 154, 1-15.

Hepler, P. K. (1997). Tip growth in pollen tubes: calcium leads the way. Trends Plant Sci. 2, 79-80.

Honys, D., Renak, D., and Twell, D. (2006). "Male gametophyte development and function," in Floriculture, Ornamental and Plant Biotechnology: Advances and Topical Issues, ed. J. Teixera da Silva (London: Global Science Books), 76-87.

Hoson, T., Matsumoto, S., Soga, K., and Wakabayashi, K. (2010). Cortical microtubules are responsible for gravity resistance in plants. Plant Signal. Behav. 5, 752-754.

Hoson, T., Nishitani, K., Miyamoto, K., Ueda, J., Kamisaka, S., Yamamoto, R., and Masuda, Y. (1996). Effects of hypergravity on growth and cell wall properties of cress hypocotyls. J. Exp. Bot. 47, 513-517.

Hoson, T., and Soga, K. (2003). New aspects of gravity responses in plant cells. Int. Rev. Cytol. 229, 209-244.

Hou, G., Kramer, V. L., Wang, Y.-S., Chen, R., Perbal, G., Gilroy, S., and Blancaflor, E. B. (2004). The promotion of gravitropism in Arabidopsis roots upon actin disruption is coupled with the extended alkalinization of the columella cytoplasm and a persistent lateral auxin gradient. Plant J. 39, 113-125.

Hou, G., Mohamalawari, D. R., and Blancaflor, E. B. (2003). Enhanced gravitropism of roots with a disrupted cap actin cytoskeleton. Plant Physiol. 131, 1360-1373.

Hush, J. M., and Overall, R. L. (1991). Electrical and mechanical fields orient cortical microtubules in higher plant tissues. Cell Biol. Int. Rep. 15, 551-560.

Ingber, D. (2006). Cellular mechanotransduction: putting all the pieces together again. FASEB J. 20, 11-27.

Ingber, D. E. (1993). Cellular tensegrity: defining new rules of biological design that govern the cytoskeleton. J. Cell Sci. 104, 613-627.

Ingber, D. E. (1999). How cells (might) sense microgravity. FASEB J. 13, 3-15.

Iversen, T.-H., Johnsson, A., Skagen, E., Ødegaard, E., Beisvåg, T., Chinga, G., Andreassen, P., Wold, A., Kittang, A.I., Hammervold, A., and Rasmussen, O. (1999). Effect of a microgravity environment and influences of variations in gravity on the regeneration of rapeseed plant protoplasts flown on the S/MM-03 mission. ESASP 1222, 103-117.
Kiss, J. Z. (2000). Mechanisms of the early phases of plant gravitropism. CRC Crit. Rev. Plant Sci. 19, 551-573.

Kordyum, E. L. (2003). Calcium signaling in plant cells in altered gravity. Adv. Space Res. 32, 1621-1630.

Kroeger, J. H., Bou Daher, F., Grant, M., and Geitmann, A. (2009). Microfilament orientation constrains vesicle flow and spatial distribution in growing pollen tubes. Biophys. J. 97, 1822-1831.

Kuang, A., Musgrave, M. E., and Matthews, S. W. (1996). Modification of reproductive development in Arabidopsis thaliana under spaceflight conditions. Planta 198, 588-594.

Kuang, A., Musgrave, M. E., Matthews, S. W., Cummins, D. B., and Tucker, S. C. (1995). Pollen and ovule development in Arabidopsis thaliana under spaceflight condition. Am. J. Bot. 82, 585-595.

Kuang, A., Popova, A., McClure, G., and Musgrave, M. E. (2005). Dynamics of storage reserve deposition during Brassica rapa L. pollen and seed development in microgravity. Int. J. Plant Sci. 166, 85-96.

Kuznetsov, O., Brown, C., Levine, H., Piastuch, W., Sanwo-Lewandowski, M., and Hasenstein, K. (2001). Composition and physical properties of starch in microgravity-grown plants. Adv. Space Res. 28, 651-658.

Kuznetsov, O. A., and Hasenstein, K. H. (1996). Intracellular magnetophoresis of amyloplasts and induction of root curvature. Planta 198, 87-94.

Kuznetsov, O. A., and Hasenstein, K. H. (1997). Magnetophoretic induction of curvature in coleoptiles and hypocotyls. J. Exp. Bot. 48, 1951-1957.

Kuznetsov, O. A., and Hasenstein, K. H. (2001). Intracellular magnetophoresis of statoliths in Chara rhizoids and analysis of cytoplasm viscoelasticity. Adv. Space Res. 27, 887-892.

Leitz, G., Kang, B., Schoenwaelder, M. E. A., and Staehelin, L. A. (2009). Statolith sedimentation kinetics and force transduction to the cortical endoplasmic reticulum in gravitysensing Arabidopsis columella cells. Plant Cell 21, 843-860.

Levine, L., Heyenga, A., Levine, H., Choi, J., Davin, L., Krikorian, A., and Lewis, N. (2001). Cell-wall architecture and lignin composition of wheat development in a microgravity environment. Phytochemistry 57, 835-846.

Lewis, M., Reynolds, J., Cubano, L., Hatton, J., Lawless, B., and Piepmeier, E. (1998). Spaceflight alters microtubules and increases apoptosis in human lymphocytes (Jurkat). FASEB J. 12, 1007-1018.
Limbach, C., Hauslage, J., Schäfer, C. and Braun, M. (2005). How to activate a plant gravireceptor. Early mechanism of gravity sensing studied in characean rhizoids during parabolic flights. Plant Physiol. 139, 1030-1040.

Lisboa, Y. S., Scherer, G. E. F., and Quader, H. (2002). Endocytosis in tobacco pollen tubes: visualisation and measurement of plasma membrane retrieval during different gravity conditions indicates gravitydependence of endocytosis. J. Gravit. Physiol. 9, P239-P240.

Liu, B., Ho, C.-M. K., and Lee, Y.-R J. (2011). Microtubule reorganization during mitosis and cytokinesis: lessons learned from developing microgametophytes in Arabidopsis thaliana. Front. Plant Sci. 2:27. doi: 10.3389/fpls.2011.00027

Luttges, M. (1992). Recognizing and optimizing flight opportunities with hardware and life sciences limitations. Trans. Kans. Acad. Sci. 95, 76-86.

Martzivanou, M., Babbick, M., CogoliGreuter, M., and Hampp, R. (2006). Microgravity-related changes in gene expression after short-term exposure of Arabidopsis thaliana cell cultures. Protoplasma 229, 155-162.

Martzivanou, M., and Hampp, R. (2003). Hyper-gravity effects on the Arabidopsis transcriptome. Physiol. Plant. 118, 221-231.

Matsumoto, S., Kumasaki, S., Soga, K., Wakabayashi, K., Hashimoto, T., and Hoson, T. (2010). Gravityinduced modifications to development in hypocotyls of Arabidopsis tubulin mutants. Plant Physiol. 152, 918-926.

Matsumoto, S., Saito, Y., Kumasaki, S., Soga, K., Wakabayashi, K., and Hoson, T. (2007). Upregulation of tubulin genes and roles of microtubules in hypergravity induced growth modifications in Arabidopsis hypocotyls. Adv. Space Res. 39, 1176-1181.

Morita, M. T. (2010). Directional gravity sensing in gravitropism. Annu. Rev. Plant Biol. 61, 705-720.

Morita, M. T., and Tasaka, M. (2004). Gravity sensing and signaling. Curr. Opin. Plant Biol. 7, 712-718.

Mullen, J., Wolverton, C., Ishikawa H., and Evans, M. (2000). Kinetics of constant gravitropic stimulus responses in Arabidopsis roots using a feedback system. Plant Physiol. 123, 665-670.

Musgrave, M. (2007). Growing plants in space. CAB Rev. Perspect. Agric. Vet. Sci. Nutr. Nat. Resour. 2, 65.

Musgrave, M., Kuang, A., Allen, J. Blasiak, J., and van Loon, J. J. W. A. (2009a). Brassica rapa L. seed development in hypergravity. Seed Sci. Res. 19, 63-72.

Musgrave, M., Kuang, A., Allen, J. and van Loon, J. J. W. A. (2009b). Hypergravity prevents seed production in Arabidopsis by disrupting pollen tube growth. Planta 230, 863-870.

Musgrave, M. E., Kuang, A., and Matthews, S. W. (1997). Plant reproduction during spaceflight: importance of the gaseous environment. Planta 203, S177-S184.

Nedukha, E. M. (1996). Possible mechanisms of plant cell wall changes at microgravity. Adv. Space Res. 17, 37-45.

Nedukha, E. M. (1998). Effects of clinorotation on the polysaccharide content of resynthesized walls of protoplasts. Adv. Space Res. 21, 1121-1126.

Nedukha, E. M., Sidorov, V. A., and Samoylov, V. M. (1994). Clinostation influence on regeneration of a cell wall in Solanum tuberosum L. protoplasts. Adv. Space Res. 14, 97-101.

Orr, A., Helmke, B., Blackman, B., and Schwartz, M. (2006). Mechanisms of mechanotransduction. Dev. Cell 10, 11-20.

Papaseit, C., Pochon, N., and Tabony, J. (2000). Microtubule selforganization is gravity-dependent. Proc. Natl. Acad. Sci. U.S.A. 97, 8364-8368.

Perbal, G., and Driss-Ecole, D. (2003). Mechanotransduction in gravisensing cells. Trends Plant Sci. 8, 498-504.

Poirier, C., and Iglesias, P. (2007). An integrative approach to understanding mechanosensation. Brief. Bioinform. 8, 258-265.

Popova, A., Musgrave, M., and Kuang, A. (2009). The development of embryos in Brassica rapa L. in microgravity. Cytol. Genet. 43, 89-93.

Rashotte, A. M., Brady, S. R., Reed, R. C., Ante, S. J., and Muday, G. K. (2000). Basipetal auxin transport is required for gravitropism in roots of Arabidopsis. Plant Physiol. 122, 481-490.

Rasmussen, O., Baggerud, C. A., Larssen, H. C., Evjen, K., and Iversen, T.H. (1994). The effect of 8 days of microgravity on regeneration of intact plants from protoplasts. Physiol. Plant. 92, 404-411.

Rasmussen, O., Klymchuk, D. A., Kordyum, E. L., Danevich, L. A., Tarnavskaya, E. B., Lozovaya, V. V., Tairbekov, M. G., Baggemd, C., and Iversen, T.-H. (1992). The effect of exposure to microgravity on the development and structural organization of plant protoplasts flown 
on Biokosmos 9. Physiol. Plant. 84, 162-170.

Rösner, H., Wassermann, T., Möller, W., and Hanke, W. (2006). Effects of altered gravity on the actin and microtubule cytoskeleton of human SH-SY5Y neuroblastoma cells. Protoplasma 229, 225-234.

Roux, S. J., Chatterjee, A., Hillier, S., and Cannon, T. (2003). Early development of fern gametophytes in microgravity. Adv. Space Res. 31, 215-220.

Sack, F. D. (1997). Plastids and gravitropic sensing. Planta 203, S63-S68.

Salmi, M., ul Haque, A., Bushart, T., Stout, S., Roux, S., and Porterfield, D. (2011). Changes in gravity rapidly alter the magnitude and direction of a cellular calcium current. Planta 233, 911-920.

Sato, F., Takeda, S., Matsushima, H., and Yamada, Y. (1999). Cell growth and organ differentiation in cultured tobacco cells under spaceflight condition. Biol. Sci. Space 13, 18-24.

Schwuchow, J., Sack, F. D., and Hartmann, E. (1990). Microtubule distribution in gravitropic protonemata of the moss Ceratodon protoplasma 159, 60-69.

Schwuchow, J. M., Kim, D., and Sack, F. D. (1995). Caulonemal gravitropism and amyloplast sedimentation in the moss Funaria. Can. J. Bot. 73, 1029-1035.

Sieberer, B., Kieft, H., FranssenVerheijen, T., Emons, A., and Vos, J. (2009). Cell proliferation, cell shape, and microtubule and cellulose microfibril organization of tobacco BY-2 cells are not altered by exposure to near weightlessness in space. Planta 230, 1129-1140.

Sievers, A., Buchen, B., and Hodick, D. (1996). Gravity sensing in tipgrowing cells. Trends Plant Sci. 1, 273-279.

Sinclair, W., and Trewavas, A. J. (1997). Calcium in gravitropism: a reexamination. Planta 203, S85-S90.

Skagen, E., and Iversen, T.-H. (1999). Simulated weightlessness and hyper-g results in opposite effects on the regeneration of the cortical microtubule array in protoplasts from Brassica napus hypocotyls. Physiol. Plant. 106, 318-325.
Skagen, E., and Iversen, T.-H. (2000). Effect of simulated and real weightlessness on early regeneration stages of Brassica napus protoplasts. In vitro Cell. Dev. Biol. Plant 36, 312-318.

Soga, K., Harada, K., Wakabayashi, K., Hoson, T., and Kamisaka, S. (1999a). Increased molecular mass of hemicellulosic polysaccharides is involved in growth inhibition of maize coleoptiles and mesocotyls under hypergravity conditions. $J$. Plant Res. 112, 273-278.

Soga, K., Wakabayashi, K., Hoson, T., and Kamisaka, S. (1999b). Hypergravity increases the molecular size of xyloglucans by decreasing xyloglucan-degrading activity in azuki bean epicotyls. Plant Cell Physiol. 40, 581-585.

Soga, K., Wakabayashi, K., Hoson, T., and Kamisaka, S. (2001). Gravitational force regulates elongation growth of Arabidopsis hypocotyls by modifying xyloglucan metabolism. Adv. Space Res. 27, 1011-1016.

Soga, K., Wakabayashi, K., Kamisaka, S., and Hoson, T. (2002). Perception of gravity stimuli by mechanosensitive ion channels in plant seedlings. Plant Cell Physiol. 43, s182.

Soga, K., Wakabayashi, K., Kamisaka, S., and Hoson, T. (2005). Mechanoreceptors rather than sedimentable amyloplasts perceive the gravity signal in hypergravity-induced inhibition of root growth in azuki bean. Funct. Plant Biol. 32, 175-179.

Soga, K., Wakabayashi, K., Kamisaka, S., and Hoson, T. (2006). Hypergravity induces reorientation of cortical microtubules and modifies growth anisotropy in azuki bean epicotyls. Planta 224, 1485-1494.

Staves, M. P. (1997). Cytoplasmic streaming and gravity sensing in Chara internodal cells. Planta 203, S79-S84.

Tamaoki, D., Karahara, I., Nishiuchi, T., Wakasugi, T., Yamada, K., and Kamisaka, S. (2011). Involvement of auxin dynamics in hypergravityinduced promotion of lignin-related gene expression in Arabidopsis inflo- rescence stems. J. Exp. Bot. doi: 10.1093/jxb/err224. [Epub ahead of print].

Toyota, M., Furuichi, T., Tatsumi, H., and Sokabe, M. (2008). Cytoplasmic calcium increases in response to changes in the gravity vector in hypocotyls and petioles of Arabidopsis seedlings. Plant Physiol. 146, 505-514.

Volkmann, D., and Baluska, F. (2006). Gravity: one of the driving forces for evolution. Protoplasma 229, 143-148.

Waldron, K., and Brett, C. (1990). Effects of extreme acceleration on the germination, growth and cell wall composition of pea epicotyls. J. Exp. Bot. 41, 71-77.

Wang, H., Zheng, H. Q., Sha, W. Zeng, R., and Xia, Q. C. (2006). A proteomic approach to analysing responses of Arabidopsis thaliana callus cells to clinostat rotation. $J$. Exp. Bot. 57, 827-835.

Wayne, R., and Staves, M. P. (1996). A down to earth model of gravisensing of Newton's Law of Gravitation from the apple's perspective. Physiol. Plant. 98, 917-921.

Wayne, R., Staves, M. P., and Leopold, A C. (1992). The contribution of the extracellular matrix to gravisensing in characean cells. J. Cell Sci. 101, 611-623.

Weise, S., Kuznetsov, O. A., Hasenstein, K. H., and Kiss, J. Z. (2000). Curvature in Arabidopsis inflorescence stems is limited to the region of amyloplast displacement. Plant Cell Physiol. 41, 702-709.

Wheeler, R. M. (2010). Plants for human life support in space: from Myers to Mars. Gravit. Space Biol. 23, 25-34.

Williams, D. (2002). "Isolation and integrated testing: an introduction to the Lunar-Mars life support test project," in Isolation: NASA Experiments in Closed-Environment Living, eds H. Lane, R. Sauer, and D. Feeback (San Diego: Univelt), 1-6.

Wolverton, C., and Kiss, J. Z. (2009). An update on plant space biology. Gravit. Space Biol. Bull. 22, 13-20.

Wolverton, C., Mullen, J., Ishikawa, H., and Evans, M. (2002). Root gravit- ropism in response to a signal originating outside of the cap. Planta 215, 153-157.

Wu, H.-M., Hazak, O., Cheung, A. Y., and Yalovsky, S. (2011). RAC/ROP GTPases and auxin signaling. Plant Cell 23, 1208-1218.

Yamamoto, R., and Kiss, J. Z. (2002). Disruption of the actin cytoskeleton results in the promotion of gravitropism in inflorescence stems and hypocotyls of Arabidopsis. Plant Physiol. 28, 669-681.

Yang, C., Wei, D., and Zhuang, F. Y. (2008). The force induced by organelles' weight in the microfilament is in the range of $0.1-1 \mathrm{pN}$. Acta Astronaut. 63, 923-928.

Zhao, Y. (2010). Auxin biosynthesis and its role in plant development. Annu. Rev. Plant Biol. 61, 49-64.

Zheng, H.-Q., and Staehelin, L. A. (2001). Nodal endoplasmic reticulum, a specialized form of endoplasmic reticulum found in gravity-sensing root tip columella cells. Plant Physiol. 125, 252-265.

Zimmermann, R. (2003). Growing pains. Air Space 31-35.

Conflict of Interest Statement: The authors declare that the research was conducted in the absence of any commercial or financial relationships that could be construed as a potential conflict of interest.

Received: 04 June 2011; accepted: 05 September 2011; published online: 28 September 2011.

Citation: Chebli $Y$ and Geitmann A (2011) Gravity research on plants: use of single-cell experimental models. Front. Plant Sci. 2:56. doi: 10.3389/fpls.2011.00056

This article was submitted to Frontiers in Plant Cell Biology, a specialty of Frontiers in Plant Science.

Copyright (C) 2011 Chebli and Geitmann. This is an open-access article subject to a non-exclusive license between the authors and Frontiers Media SA, which permits use, distribution and reproduction in other forums, provided the original authors and source are credited and other Frontiers conditions are complied with. 